\title{
AVALIAÇÃO DE MODELOS GLOBAIS DO GEOPOTENCIAL PARA OS ESTADOS DO MARANHÃO E PIAUÍ
}

\author{
Evaluation of Global Geopotential Models for the States of Maranhão and Piaui \\ LINEARDO FERREIRA DE SAMPAIO MELO ${ }^{1}$ \\ SÍLVIO ROGÉRIO CORREIA DE FREITAS ${ }^{1}$ \\ ${ }^{1}$ Programa de Pós-Graduação em Ciências Geodésicas \\ Universidade Federal do Paraná - Curitiba - Brasil \\ lineardo@yahoo.com.br; sfreitas@ufpr.br
}

\begin{abstract}
RESUMO
A avaliação do modelo geoidal oficial do Brasil, o MAPGEO2010, mostra que suas menores resoluções situam-se nos estados do Maranhão e do Piauí, com discrepâncias variando de cerca de $\pm 1 \mathrm{~m}$ em $500 \mathrm{~km}$. Justamente, em tal região se faz necessário um melhor modelo geoidal em vista das limitações de informações geodésicas existentes. Com base no problema evidenciado na região destacada, buscou-se avaliar o comportamento das alturas geoidais oriundas de alguns Modelos Globais do Geopotencial MGGs. Foram utilizados como base para a avaliação, dados do Sistema Geodésico Brasileiro (SGB) de Estações Geodésicas (EG) onde foram realizadas observações GPS da altitude elipsóidica sobre Referências de Nível da Rede Altimétrica Fundamental do Brasil (RAFB), aqui denominadas de EG-GPS/NIV . Os MGGs empregados são: satélite somente das missões GRACE e GOCE, e os modelos combinados EIGEN-05C no grau 360 e EGM2008 nos graus 360,720 e 2.190. Os modelos combinados têm por base dados de satélites (GRACE, Lageos), altimetria e gravimetria de diversas bases de dados. Neste estudo foram selecionadas 47 EG - GPS/NIV do IBGE distribuídas na região compreendida entre as longitudes $-47,5^{\circ} \leq \lambda \leq-41^{\circ}$ e latitudes $-10^{\circ} \leq \varphi \leq-3^{\circ}$. Para cada estação foi calculado o valor da altura geoidal a partir de diversos MGGs bem como o MAPGEO2010. Com base nas análises efetivadas conclui-se que existem modelos globais com boa adequação na região de estudos.

Palavras-chave: Modelo Global do Geopotencial - MGG; Missões Gravimétricas Espaciais; Sistema Geodésico Brasileiro - SGB; MAPGEO2010.
\end{abstract}




\section{ABSTRACT}

The evaluation of the official geoid model of Brazil, MAPGEO2010, shows that the lowest model resolutions are in the States of Maranhão and Piauí with discrepancies ranging from -1 to $+1 \mathrm{~m}$ in about $500 \mathrm{~km}$. Just for these regions, it is necessary an accurate geoid model in view of the limitations of existing geodetic information. In view of the problem evidenced in the highlighted region we carried out the evaluation of the geoid heights derived from some Global Geopotential Models (GGMs). Data from the Brazilian Geodetic System (BGS) comprising bench marks of Brazilian Fundamental Altimetric Network (RAFB) were GPS ellipsoidal heights were determined (EG-GPS/NIV). The employed GGMs came from satellite-only (from GRACE and GOCE missions) and the combined GGMs EGM2008 developed up to the degrees, 360, 720 and 2190 and EIGEN_05C in the degree and order 360, both with satellite data from GRACE and Lageos missions, as well as altimetry and gravimetry from several data bases. For the study, 47 geodetic stations (EGGPS/NIV) of Brazilian Institute of Geography and Statistics (IBGE) were selected and distributed in the study area comprised among longitude $-47,5^{\circ} \leq \lambda \leq-41^{\circ}$ and latitude $-10^{\circ} \leq \varphi \leq-3^{\circ}$. For each station it was calculated the value of the geoid height from the various GGMs. Based on the analysis presented it follows that there are global models with good fit in the region of studies.

Keywords: Global Geopotential Model - GGM; Space Gravity Missions; Brazilian Geodetic System - BGS; MAPGEO2010.

\section{INTRODUÇÃO}

\subsection{Considerações Iniciais}

Desde o início da era espacial, as investigações e modelagem do campo gravitacional da Terra têm sido conduzidas com sucesso por meio de observações das perturbações nas órbitas dos satélites em baixa altitude. Estas perturbações são induzidas pelo efeito gravitacional de heterogeneidades de massas relativamente a uma distribuição esférica homogênea, gerando informações das anomalias associadas às feições de longos e médios comprimentos de onda deste campo. Estas informações associadas ao potencial centrífugo possibilitam o estabelecimento de modelos do geopotencial ( $c f$. KAULA, 1966).

As heterogeneidades do campo gravitacional, associadas distribuições anômalas de massa, é usada na determinação da figura da Terra. Um exemplo de aplicação é a determinação de superfícies equipotenciais, em particular, a determinação do geóide. O geoide, de acordo com a definição dada por Gauss e Listing em 1860 (apud BURŠA et al, 1999), é entendido como a superfície equipotencial melhor ajustada, no sentido dos princípios do Método dos Mínimos Quadrados (MMQ), ao nível médio do mar, avaliado globalmente e suposto este em repouso. Tal condição pode ser expressa por (BURŠA et al, 1999): 


$$
\int_{\sigma}\left(W-W_{0}\right)^{2} d \sigma=\min
$$

onde a integral é realizada sobre toda a superfície do mar, $W$ é o geopotencial avaliado em cada elemento de área $d \sigma$ e $W_{0}$ é o valor do geopotencial para o geóide. Esta definição é entendida na atualidade como a definição oceanográfica do geoide. $W$ pode ser determinado sobre os oceanos, e.g., via altimetria por satélites e $W_{0}$ é usualmente adotado como igual ao esferopotencial do modelo Terra normal com base no elipsóide de referência ou determinado, como é possível na atualidade, via integração de missões satelitais dedicadas ao campo gravitacional e Satellite Laser Ranging - SLR (DE FREITAS et al., 2007).

O grau de desenvolvimento das missões de gravimetria por satélites, a melhor modelagem da superfície física da Terra possibilitada pelo Global Navigation Satellite System (GNSS) e a atual disponibilidade de dados advindos de bases globais, tais como modelos digitais de elevação (MDEs), permite que novas pesquisas sobre as componentes do campo de gravidade da Terra sejam desenvolvidas para áreas sem adequada base de dados geodésicos convencionais. Estas perspectivas já foram exploradas por Jamur e de Freitas (2008) relativamente aos primeiros MGGs combinados com informações advindas das missões CHAllenging Mini-Satellite Payload (CHAMP) e Gravity Recovery and Climate Experiment (GRACE) e também antevendo os impactos da missão Gravity Field and Steady-State Ocean Circulation Explorer (GOCE).

Neste trabalho são analisadas as alturas geoidais dos MGGs bem como avaliadas as alturas geoidais oficiais do Sistema Geodésico Brasileiro (SGB), expressas no Modelo de Ondulação Geoidal MAPGEO2010 (IBGE, 2010). Os MGGs empregados neste trabalho são parte oriundos somente de satélites (derivados das missões gravimétricas GOCE e GRACE) e de MGGs combinados oriundos de análise orbital de satélites laser, gravimetria por satélites, gravimetria terrestre, altimetria por satélites e MDEs. A base de avaliação consiste na diferença entre altitudes elipsoidais obtidas com o Global Positioning System (GPS), o principal sistema em uso no Brasil associado ao GNSS sobre referências de nível (RNs) da RAFB, denominados aqui de EG-GPS/NIV.

\subsection{Altitude Ortométrica}

O geóide difere da superfície média dos oceanos por uma grandeza linear designada por Topografia do Nível Médio do Mar - TNMM (DE FREITAS et al., 2007). Sua altura relativamente ao elipsóide de referência, denominada de altura geoidal, é expressa como:

$$
N \cong h-H
$$


onde $h$ é a altitude elipsoidal, avaliada ao longo da normal ao elipsóide e $H$ a altitude ortométrica, avaliada ao longo da linha de campo da gravidade. $\mathrm{Na}(02)$ a consideração de colinearidade desses dois tipos de altitude não traz prejuízo perceptível ao cálculo de $N$ (JEKELI, 2000).

No cálculo da altitude ortométrica, o valor da gravidade deve ser o valor médio $(g)$ entre o geóide $P_{0}$ e o ponto $P$, ao longo da linha de campo, ou seja, (HEISKANEN \& MORITZ, 1979):

$$
H^{O}=\frac{1}{\bar{g}} \int_{P_{0}}^{P} g \cdot d n \approx \frac{1}{\bar{g}} \sum_{i=1}^{n} g_{i} \cdot d n_{i}
$$

Na prática a integral (03) deve ser substituída por um somatório sobre um conjunto discreto de valores médios da gravidade entre pontos nos quais foram observados valores de gravidade e desníveis.

Altura geoidal é obtida de forma convencional com base na solução do Problema de Valor de Contorno da Geodésia (PVCG), tal como possibilitado pela Equação de Pizetti-Stokes e da Equação de Bruns (HEISKANEN \& MORITZ, 1967, pp 53).

Cabe ser destacado que as altitudes da RAFB estão estruturadas em um sistema de altitudes ortométricas-normais, incorporando somente correções teóricas do campo da gravidade. As altitudes ortométricas-normais constituem-se em um sistema de altitudes sem o pleno significado físico tal como encontrado por exemplo nas altitudes ortométricas relacionadas a uma superfície de referência equipotencial (geoide) e mesmo nas altitudes normais relacionadas ao quase-geóide, não equipotencial porém que pode ser relacionado ao geóide.

\subsection{MGGs Atuais e o MAPGEO2010}

A popularização do GNSS, em particular do GPS, revolucionou as atividades que de posicionamento em função de sua rapidez e precisão na obtenção de coordenadas. Este fato gerou um crescente interesse por um geóide mais acurado e preciso para as diversas aplicações na área da Geodésia, onde há necessidade do conhecimento de uma altitude com significado físico. Para que as altitudes elipsoidais, oriundas de levantamentos com GNSS, possam ser utilizadas nestas áreas, é necessário que elas sejam convertidas em altitudes ortométricas, referidas ao geóide. Para isso, necessita-se da altura geoidal, ou seja, a separação entre as duas superfícies de referência, o geóide e o elipsóide, $c f$. a relação (02). Um conjunto de MGGs atuais, oriundos de dados da Missão GRACE e algumas vezes associados com dados oriundos de Satellite Laser Ranging (SLR) da missão Lageos bem como com dados da missão CHAMP causaram grande impacto nos estudos do campo gravitacional terrestre e sua variação temporal. Estes MGGs satélite somente atingiram resoluções espaciais no intervalo $1000 \mathrm{~km} \leq \lambda / 2 \leq 200 \mathrm{~km}$. O maior impacto foi o da consistência global das informações em um mesmo referencial. 
Estes MGGs tornaram-se fundamentais no monitoramento de mudanças globais associadas ao fluxo de massas e variações temporais associadas ao campo gravitacional terrestre (DE FREITAS, 2010).

Uma resolução espacial de cerca de $200 \mathrm{~km}$ é considerada ainda baixa para a geração de geóides regionais para aplicações em atividades convencionais de engenharia. Porém, atualmente existem técnicas que combinam dados locais (e.g., anomalias da gravidade) com dados globais. Já os MGGs de alta-resolução são mais adequados nas aplicações onde seja necessário um conhecimento preciso do potencial de gravidade estático e seus gradientes no espectro de comprimentos de onda médio e curto. Como exemplo, cita-se: a determinação da órbita precisa de satélites geodésicos e altimétricos, para a predição de grandezas gravimétricas, modelos geoidais com resolução na ordem de decímetros, parametrização da deflexão da vertical para vínculo de levantamentos topográficos aos efetivados com GNSS em referencial global,, etc.. No entanto, em geral, são afetados por inconsistências de referenciais associados às grandezas combinadas.

Os MGGs com dados da missão GOCE e alguns com associação a dados da missão GRACE, começaram a ser divulgados em 2010. Eles vieram impactar positivamente a Geodésia no que diz respeito a um melhor conhecimento do campo gravitacional. Vieram a se inserir em uma resolução espacial de até $80 \mathrm{~km}$, porém sem inconsistências de referencial que afetam os modelos combinados. Com isto a comunidade geodésica tem amplas expectativas nas potencialidades destes modelos para aplicações em engenharia, tal como será abordado na seqüência.

A missão GOCE, atualmente determina anomalias da gravidade, alturas geoidais entre outras componentes. A missão tem como objetivos: (DRINKWATER et al., 2007):

$\checkmark$ Medição do campo da anomalia da gravidade terrestre com uma precisão melhor do que 1 - $2 \mathrm{mGal}(1 \mathrm{mGal}=10-5 \mathrm{~ms}-2)$ a partir de combinação do gradiente da gravidade e de monitoramento de satélite - satélite;

$\checkmark$ Determinar o geóide, com uma precisão melhor do que $1-2 \mathrm{~cm}$;

$\checkmark$ Atingir as medições em uma escala de comprimento de $100 \mathrm{~km}$, ou menos (ou seja, grau e ordem igual ou superior a 200 na expansão de harmônicos esféricos do campo). Na atualidade os modelos já atingem a grau e ordem 250.

O modelo EGM2008 (PAVLIS et al., 2008) é tido, na atualidade, como o melhor resultado obtido em termos de combinação de dados gravimétricos e altimétricos oriundos de diferentes fontes. Tem sido apontado como uma das melhores estruturas de dados para a formação de um Sistema Global de Altitudes (SGA) (cf., REFAG2010, 2011). No entanto, devem-se considerar as inconsistências do geopotencial associada a cada datum vertical e particularidades das redes de nivelamento, tal como já realizado por Ferreira (2008) para o Datum Vertical Brasileiro (DVB) relativamente ao EGM96 (LEMOINE et al., 1998) e por Palmeiro (2011) para o EGM2008. O EGM2008 está completo até o grau 2.190 e ordem 2.159 em termos dos coeficientes do geopotencial, com resolução espacial de 
aproximadamente $9 \mathrm{~km}$ para o campo de gravidade em todo o globo. O objetivo do modelo EGM2008 é atingir uma acurácia global do geóide com RMS melhor que $\pm 15 \mathrm{~cm}$. Para isso, um grande esforço foi despendido pelo National GeospatialIntelligence Agency (NGA) para reunir o máximo de dados possível que pudesse auxiliar no desenvolvimento desse novo modelo. Foram utilizadas as melhores fontes de dados gravimétricos mundiais disponíveis contendo gravimetria terrestre, marinha e aérea bem como dados de, missões satelitais. Os principais auxiliares nessa tarefa foram os satélites da missão GRACE (ICGEM, 2010).

O MGG EIGEN 05C foi divulgado no ano 2008. Está completo até o grau 360 em termos dos coeficientes do geopotencial. Bastante semelhante ao modelo EIGEN GL 04C, também é composto da combinação de missões satelitais (GRACE, Lageos), dados altimétricos (MDEs e altimetria por satélite) e gravimétricos da superfície. Na Tabela 1 estão os modelos utilizados neste estudo.

Tabela 1 - Modelos globais do geopotencial utilizados.

\begin{tabular}{|c|c|c|c|c|}
\hline Modelo & Ano & Grau & Dados & Referências \\
\hline AIUB-GRACE03S & 2011 & 160 & S(GRACE) & $\begin{array}{l}\text { Jäggi et al., } \\
2011\end{array}$ \\
\hline $\begin{array}{l}\text { GO_CONS_GCF_2_TIM_ } \\
\text { R2 }\end{array}$ & 2011 & 250 & $\mathrm{~S}(\mathrm{GOCE})$ & $\begin{array}{l}\text { Pail et al., } \\
2011\end{array}$ \\
\hline EIGEN-5C & 2008 & 360 & $\begin{array}{c}\text { S(GRACE,Lageos), } \\
\text { G, A }\end{array}$ & $\begin{array}{l}\text { Förste et al., } \\
2008\end{array}$ \\
\hline \multirow{3}{*}{ EGM2008 } & \multirow{3}{*}{2008} & 2190 & \multirow{3}{*}{$\mathrm{S}$ (GRACE), G, A } & \multirow{3}{*}{$\begin{array}{l}\text { Pavlis et al., } \\
\quad 2008\end{array}$} \\
\hline & & 720 & & \\
\hline & & 360 & & \\
\hline
\end{tabular}

Nota: As letras S, G e A, na coluna de dados, significam a fonte de dados dos MGGs, sendo respectivamente Satélites, Gravimetria e Altimetria (nos continentes e regiões costeiras vinda dos MDEs e nas regiões oceânicas por altimetria por satélite).

O MAPGEO2010, abrange área compreendida pelas latitudes de $6^{\circ} \mathrm{N}$ e $35^{\circ} \mathrm{S}$ e pelas longitudes de $75^{\circ} \mathrm{W}$ e $30^{\circ} \mathrm{W}$. Utiliza o MDE SAM3s_v2, o qual baseia-se no Shuttle Radar Topography Mission (SRTM) para a obtenção da anomalia de gravidade de Bouguer completa, do efeito topográfico direto, do efeito topográfico indireto primário, do efeito topográfico indireto secundário. As anomalias médias free-air foram determinadas em grade com resolução de $5^{\prime}$. Na região oceânica a grade foi completada com dados do modelo DNS08 obtido da altimetria por satélite pelo Danish National Space Center (DNSC) (IBGE, 2011a).

O MAPGEO2010 foi obtido pela técnica Remove-Restore com base na transformada rápida de Fourier (FFT) aplicada sobre a integral de Pizzetti-Stokes com núcleo modificado para resolução de indeterminações para curtos comprimentos de onda. O valor do termo de ordem zero utilizado pelo MAPGEO2010 é de $-41 \mathrm{~cm}$. Foi utilizado como MGG de base o EGM2008 até o 
grau e ordem 150, para remover os longos comprimentos de onda da anomalia de Helmert e para repor no final a mesma componente na altura geoidal. A avaliação deste modelo foi efetivada de forma absoluta com as alturas geoidais de 804 referências de nível que tiveram suas altitudes elipsoidais determinadas com GPS geodésico. $\mathrm{Na}$ análise relativa foram utilizados 158 pares destes pontos. Os resultados absolutos estão apresentados na Figura 1 e as análises relativas conduziram à estimativa de uma acurácia relativa de 5,8 ppm (IBGE, 2011a).

Figura 1 - Recorte de Mapa de Discrepância do MAPGEO2010 e os pontos GPS/NIV.

Fonte: IBGE, 2011.

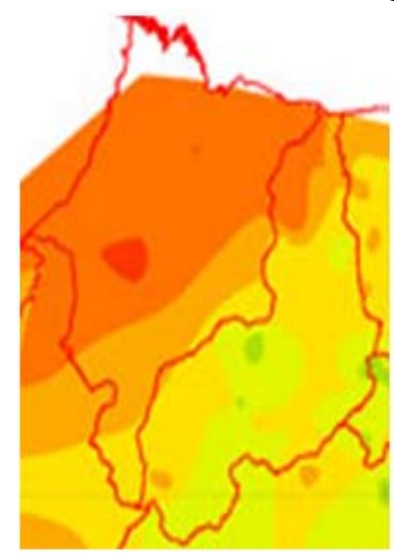

\section{Legenda}

Valor da Discrepância (m)

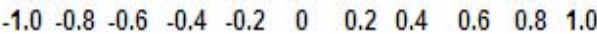

Percebe-se na Figura 1, que envolve recorte de mapa de avaliação do MAPGEO2010 (IBGE, 2011) para a área do presente estudo, que este apresenta discrepâncias na ordem de $\pm 1 \mathrm{~m}$ relativamente a EG-GPS/NIV. Também, observe-se que em parte do Estado do Maranhão não tem estimativa da acurácia do modelo.

\section{METODOLOGIA}

\subsection{Região de Estudos}

Tomando-se como referência a Região Nordeste do Brasil, com abrangência total dos estados do Maranhão e Piauí, e a base de dados disponível junto ao IBGE e do International Center for Global Gravity Field Models (ICGEM), a região de estudos é definida, conforme já referido, entre os limites de longitudes $-47,5^{\circ} \leq \lambda \leq-$ $41^{\circ}$ e latitudes $-10^{\circ} \leq \varphi \leq-3^{\circ}$ (Figura 2 ). 
Figura 2 - Localização da Área de Estudo.
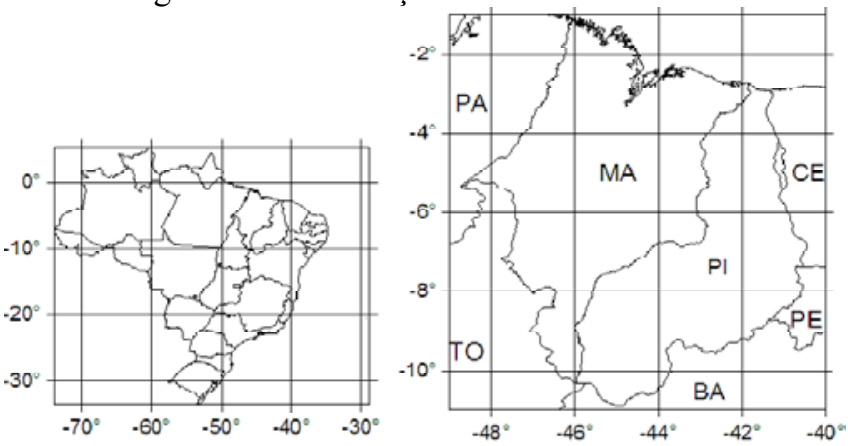

\subsection{Características dos Dados Disponíveis}

Para a estruturação da base de dados geodésicos da pesquisa, utilizaram-se as seguintes informações:

$\checkmark \quad$ Latitude, longitude e altitudes das estações geodésicas EG - GPS/NIV do banco de dados do SGB, extraídas dos dados disponíveis no sítio (http://www.ibge.gov.br/home/geociencias/geodesia/sgb.shtm).

$\checkmark$ MGGs (http://icgem.gfz-potsdam.de/ICGEM/):

$>$ EGM2008,

$>$ EIGEN 05C,

$>$ AIUB-GRACE03S e

$>$ GO_CONS_GCF_2_TIM_R2 e

$\checkmark$ Modelo de Ondülação Geoidal-MAPGEO2010.

\subsection{Dados do Sistema Geodésico Brasileiro - SGB.}

O SGB foi iniciado na década de 40. O SGB caracteriza-se pelo conjunto de estações que representam o controle horizontal e altimétrico necessários ao referenciamento espacial da informações, navegação e representação cartográfica no território brasileiro. Seu estabelecimento e manutenção são atribuições do IBGE via sua Coordenação de Geodésia. O SGB é composto pelas redes altimétrica, horizontal e gravimétrica (IBGE, 2011b).

As estações geodésicas (EG) utilizadas nesta pesquisa fazem parte da rede altimétrica. Essas estações utilizam duas metodologias para a obtenção das altitudes, sendo elas: a de monitoramento com receptores GPS para coletar o conjunto de observações das quais se pode calcular a altitude elipsoidal, e a metodologia de nivelamento geométrico desde o DVB em Imbituba - SC, para obtenção da altitude ortométrica-normal. A integração dessas duas metodologias é o que se chama de GPS/NIV, sigla esta adotada para as estações geodésicas da pesquisa. Primeiramente foram selecionadas 47 EG - GPS/NIV do banco de dados do IBGE, distribuídas na região de estudos (Figura 3). Em seguida, foram extraídas do relatório as informações de maior relevância para a pesquisa: as latitudes; as 
longitudes; as altitudes elipsoidais (fonte: GPS - Geodésico); e as altitudes ortométricas-normais (fonte: nivelamento geométrico). Posteriormente calcularamse as alturas geoidais, das 47 estações, para todos os MGGs e para o modelo de ondulação geoidal brasileiro. Seguindo o procedimento descrito anteriormente.

Figura 3 - Distribuição Geográfica das Estações Disponíveis.

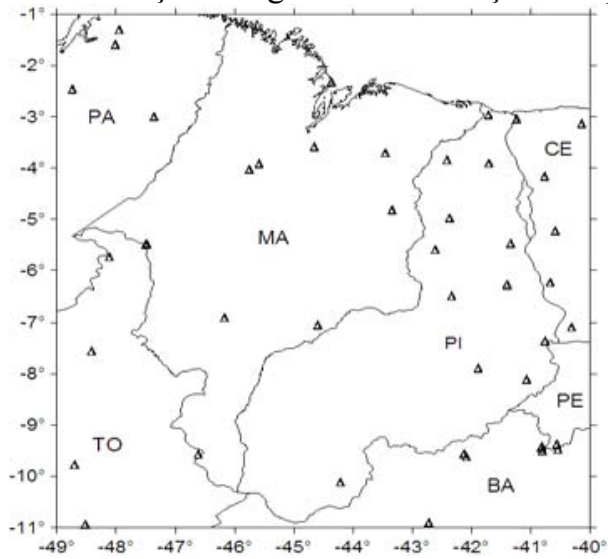

\subsection{Dados dos Modelos Globais do Geopotencial}

Os MGGs são disponibilizados on-line pelo sítio do ICGEM. O cálculo do valor da altura geoidal, dos diversos MGGs, realizou-se também em função das informações e rotinas disponíveis no sítio. Para a geração dos valores de altura geoidal, pelo sítio, é necessário disponibilizar-se as coordenadas extremas do retângulo que envolve a área de estudo. Outra alternativa é processar ponto a ponto, fornecendo a latitude e longitude de cada ponto de interesse. Para a pesquisa optouse pela metodologia de processamento ponto a ponto.

O passo seguinte foi preencher as lacunas, sendo que cada campo existem diferentes componentes, que devem ser configuradas de acordo com a finalidade do trabalho desenvolvido. Para o trabalho em questão as informações foram as seguintes:

Sistema Geodésico de Referência: no caso o utilizado foi o GRS 80 para todos os modelos, para compatibilidade e coerência das análises em SIRGAS2000, que serão explicadas na próxima seção;

$\checkmark$ Modelos a serem avaliados: foram processadas informações de todos os modelos citados anteriormente;

$\checkmark$ Função: componente a ser calculada - calculou-se a altura geoidal.

$\checkmark$ Sistema de maré permanente utilizado: tide-free;

$\checkmark \quad$ Termo do grau zero: incluído.

Para cada um dos MGGs utilizados manteve-se suas definições iniciais. Para a lacuna do sistema geodésico de referência, dentre os diversos disponibilizados (e.g. 
WGS-89, GRS-80, GRS-67 entre outros), o sistema geodésico de referência considerado na pesquisa foi o Geodetic Reference System 1980 (GRS-80), que é também o SGR de referência física e geométrica do SIRGAS2000.

Todos os cálculos efetivados nesta pesquisa foram efetuados num sistema livre de marés (tide-free), conforme as definições iniciais de cada um dos MGGs adotados (ICGEM, 2010). Também, no sítio do ICGEM é possível gerar-se os parâmetros de interesse em outros sistemas de marés. É importante deixar claro que o termo do grau zero no desenvolvimento do geopotencial em harmônicos esféricos representa o potencial de uma esfera homogênea de massa $M$ sobre um ponto a uma distância $\mathrm{R}$ do centro da mesma, sendo $\mathrm{R}$ e $\mathrm{M}$ respectivamente o raio médio da Terra e sua massa (GEMAEL, 2002).

\subsection{Dados do Modelo de Ondulação Geoidal - MAPGEO2010}

Para este cálculo empregou-se os mesmos pontos para os quais gerou-se as alturas geoidais com os MGGs considerados anteriormente. O MAPGEO2010 disponibiliza seus dados em dois sistemas de referência: SIRGAS2000 e SAD69, onde o usuário deve escolher o seu sistema de referência. Para o caso desta pesquisa utilizou-se o SIRGAS2000, o qual tem por base também o GRS80 e está no sistema de maré permanente livre de maré.

\section{RESULTADOS E DISCUSSÕES}

Nos trabalhos de Ferreira (2008), Sánchez e Martínez (2008), De Freitas et al. (2010), Ferreira (2011), entre outros, evidenciam que as diferenças entre alturas geoidais vinculadas ao DVB de Imbituba e as obtidas dos MGGs não coincide. Estes autores apresentam discrepâncias médias em torno de $-30 \mathrm{~cm}$, indicando que o DVB está abaixo da referência global. Assim, ao se considerar um geóide global para avaliação da TNMM no Datum em Imbituba obtêm-se valores aproximadamente de $-22 \mathrm{~cm} \pm 0.01 \mathrm{~m}$ (FERREIRA, 2008) $-29 \mathrm{~cm} \pm 0.01 \mathrm{~m}$ para o EGM2008 truncado no grau 360 e $-30 \mathrm{~cm} \pm 0.01 \mathrm{~m}$ para o EGM2008 no grau 2190 (DE FREITAS et al. 2010; FERREIRA, 2011).

\subsection{Avaliação Absoluta}

Em uma primeira abordagem, as avaliações foram realizadas de forma absoluta. As estatísticas dos resultados destas avaliações expressam as discrepâncias das alturas geoidais dos pontos das EG - GPS/NIV em relação às alturas geoidais advindas dos MGGs, bem como do modelo MAPGEO2010. Tais avaliações são discutidas a seguir.

Primeiramente, como se sabe que $N, H e h$, são três tipos de grandezas diferentes, mas que, no entanto, podem ser relacionadas $c f$. a (02). Logo, as diferenças entre as alturas geoidais advindas das EG - GPS/NIV e as alturas geoidais dos modelos devem atender à condição: 


$$
N_{G P S / N I V}-N_{M} \approx 0
$$

onde $N_{G P S / N I V}$ é altura geoidal dos pontos das EG - GPS/NIV e $N_{M}$ é a altura geoidal dos modelos. No entanto, devido a uma série de inconsistências, tais como ruído aleatório nos valores de $N, H$ e $h$, erros grosseiros, distorções sistemáticas, entre outras, a condição exposta na (04) não ocorre. Esse erro de fechamento, aqui se trata como diferença de altura geoidal, expressa por:

$$
\Delta N=N_{G P S / N I V}-N_{M}
$$

Aplicando-se a (05) para os 47 pontos, utilizando-se todos os MGGs já referidos, realizaram-se os cálculos estatísticos para a obtenção dos valores mínimo, máximo, médio, amplitude das variações e Erro Médio Quadrático (EQM) em relação aos dados do SGB. Destas avaliações apresentam-se os resultados na Tabela 02 .

Tabela 02 - Avaliação Absoluta dos MGGs em relação às EG - GPS/NIV do SGB.

\begin{tabular}{l|r|r|r|r|r|r}
\hline \multirow{2}{*}{ Modelos } & Mín. & Máx. & Amp. & Média & $\begin{array}{c}\text { Desv. } \\
\text { Padr. }\end{array}$ & EQM \\
\cline { 2 - 7 }$(\mathrm{m})$ \\
\hline GO_CONS_GCF_2_TIM_R2 & 0,00 & 0,96 & 0,96 & 0,30 & 0,30 & 0,42 \\
\hline AIUB-GRACE03S & 0,03 & 1,19 & 1,17 & 0,26 & 0,38 & 0,45 \\
\hline EGM2008_2190 & $-0,05$ & 0,70 & 0,75 & 0,36 & 0,20 & 0,41 \\
\hline EGM2008_720 & $-0,06$ & 0,69 & 0,75 & 0,36 & 0,19 & 0,40 \\
\hline EGM2008_360 & $-0,02$ & 0,66 & 0,68 & 0,36 & 0,19 & 0,40 \\
\hline EIGEN_05C & $-0,02$ & 1,60 & 1,61 & 0,38 & 0,34 & 0,51 \\
\hline MAPGEO2010 & $-0,06$ & $-1,08$ & $-1,02$ & $-0,52$ & 0,26 & 0,58 \\
\hline
\end{tabular}

As tendências sistemáticas dos MGGs, expressas pelos valores médios, são bastante similares e uma média destes valores resulta em $0,336 \mathrm{~m}$. Destaque-se que aqui a referência de avaliação é o SGB e, portanto, os MGGs globais têm superfície geoidal acima da referência do SGB. Já o MAPGEO2010 apresenta tendência sistemática oposta e de maior valor absoluto. Os resultados desta avaliação, em termos de valores absolutos, revelam que os modelos que apresentaram as menores componentes sistemáticas, considerando o valor médio do desvio e, portanto, uma melhor adaptação à região de estudos, foram os modelos AIUB-GRACE03S e o GO_CONS_GCF_2_TIM_R2, com $0,26 \mathrm{~m}$ e $0,30 \mathrm{~m}$ respectivamente. Os maiores desvios sistemáticos foram encontrados para os modelos MAPGEO2010 com -0,52 
m, seguido do modelo EIGEN_05C com 0,38 m. Já quando avaliado o RMS, o modelo que apresentou menor valor foi o EGM2008 com aproximadamente 0,40 m, em todos os graus de desenvolvimento avaliados e maiores RMS os modelos MAPGEO2010 com 0,58 m e EIGEN_05C com 0,51 m.

\subsection{Diferenças entre as estações advindos do GPS/NIV e dos MGGs e MAPGEO2010 \\ Os MGGs satélite somente avaliados foram o AIUB-GRACE03S e o} GO_CONS_GCF_2_TIM_R2. O primeiro advindo da missão GRACE e o segundo da missão GOCE. Estes MGGs foram desenvolvidos nos graus máximos 160 e 250 , respectivamente, apresentando resoluções espaciais de aproximadamente $125 \mathrm{~km} \mathrm{e}$ $83 \mathrm{~km}$, respectivamente. Destaque-se que estes modelos têm baixos erros de comissão e elevados erros de omissão.

Os valores das diferenças entre as EG - GPS/NIV e o MGG AIUBGRACE03S variaram em torno de $0,03 \mathrm{~m}$ a $1,19 \mathrm{~m}$ para mínimo e máximo, respectivamente, com uma amplitude de 1,17 m (Figura 4). Já para o MGG GO_CONS_GCF_2_TIM_R2 as diferenças deste modelo apresentam resultados de valor mínimo igual a $0,00 \overline{\mathrm{m}}$ e valor máximo de $0,96 \mathrm{~m}$, com amplitude de $0,96 \mathrm{~m}$ (Figura 5).

Figura 4 - Diferenças entre as EG GPS/NIV e AIUB-GRACE03S.

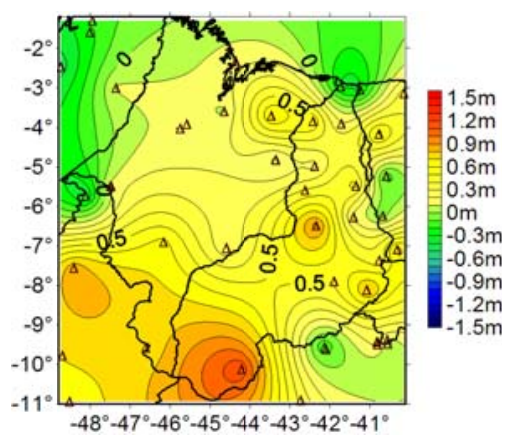

Figura 5 - Diferenças entre as EG GPS/NIV e

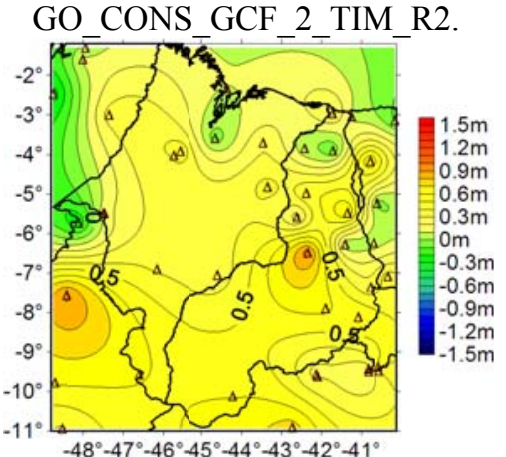

$\mathrm{Na}$ sequência, apresentam-se os MGGs EGM2008 e o EIGEN05C, da categoria combinado. O MGG EGM2008 foi avaliado em três graus de desenvolvimento, 360, 720 e no seu grau máximo 2190, com resoluções espaciais de aproximadamente $55 \mathrm{~km}, 27 \mathrm{~km}$ e $9 \mathrm{~km}$, respectivamente. Na data de realização da presente pesquisa, o EGM2008 desenvolvido em seu grau máximo era o MGG que possuía a maior resolução espacial entre os divulgados pelo ICGEM.

Para o EGM2008 truncado no grau 360, são apresentados valores próximos de zero, tem uma variação nas diferenças com as EG - GPS/NIV na ordem de $-0,02 \mathrm{~m}$ 
a 0,66 m, para valor mínimo e máximo, respectivamente, apresentando uma amplitude de $0,75 \mathrm{~m}$. Os resultados do truncamento no grau 720 apresentam o valor mínimo de $-0,06 \mathrm{~m}$ e máximo de $0,69 \mathrm{~m}$, com amplitude de $0,75 \mathrm{~m}$. Já no grau máximo 2190, o EGM2008, os resultados das diferenças entre as estações e os modelos variam em valores, mínimo e máximo, de $-0,05 \mathrm{~m}$ a $0,70 \mathrm{~m}$, respectivamente, com amplitude de $0,75 \mathrm{~m}$, conforme a Figura 6.

De uma forma geral as avaliações do EGM2008, nos três graus de desenvolvimento, apresentaram-se de maneira bastante similar. Assim, pode-se inferir que para o grau máximo (2.190) o modelo não acrescentou melhoria nas análises relativamente aos graus 360 e 720, inclusive apresentando valores ligeiramente maiores.

Na sequência apresenta-se o MGG EIGEN05C. Neste estudo trabalhou-se com este modelo desenvolvido em seu grau máximo 360. Para este grau tem-se uma resolução espacial de aproximadamente $55 \mathrm{~km}$. Com base na mesma metodologia das demais avaliações pode-se afirmar que este modelo não apresentou um bom desempenho para a região. As diferenças entre as estações e o modelo marcaram valores mínimo e máximo na faixa de $-0,02 \mathrm{~m}$ a $1,60 \mathrm{~m}$, respectivamente, com amplitude de 1,61 m (Figura 7).

Figura 6 - Diferenças entre as EG GPS/NIV e EGM2008 no grau máximo (2190).

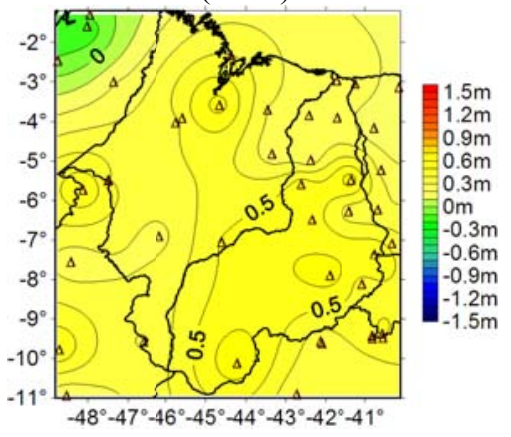

Figura 7 - Diferenças entre as EG GPS/NIV e EIGEN05C.

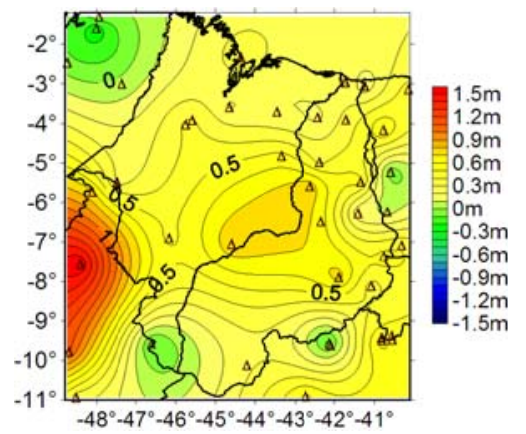

$\mathrm{Na}$ avaliação das diferenças entre o MAPGEO2010 e as estações, os valores mínimo e máximo apresentados foram de $-0,06 \mathrm{~m}$ e $-1,08 \mathrm{~m}$, respectivamente, com amplitude de $-1,02 \mathrm{~m}$, como mostra a Figura 8 . Esta diferença parece ser a maior entre todos os modelos avaliados, porém é inferior àquela apresentada nas análises de discrepância, realizada pelo IBGE, conforme o recorte de avaliação na Figura 1. A região de estudos apresenta carência de informações e prováveis deficiências na base geodésica, podendo existir erros relativos à propagação da RAFB desde Imbituba, na gravimetria no local e também no posicionamento GPS. 
Figura 8 - Diferenças entre as EG - GPS/NIV

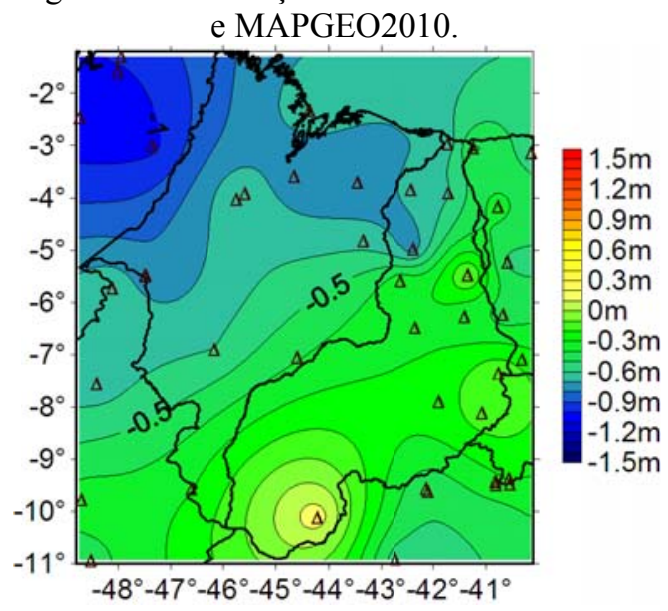

Analisando as diferenças de cada modelo, de uma forma geral, tem-se que os MGGs oriundos somente de satélite, assim como os combinados, obtiveram resultados satisfatórios, conforme já apresentado na Tabela 02. Já os resultados do MAPGEO2010 apresentam-se aparentemente destoantes nas diferenças com as EG GPS/NIV. Para essas avaliações os modelos que obtiveram os melhores resultados foram os modelos GO_CONS_GCF_2_TIM_R2, seguido do EGM2008 nos três graus e os piores resultados foram os modelos MAPGEO2010, EIGEN-05C e AIUB-GRACE03S.

\subsection{Diferença entre o MAPGEO2010 e os MGGs}

Primeiramente são apresentados os resultados dos MGGs AIUB-GRACE03S e o GO_CONS_GCF_2_TIM_R2. Tendo-se por base os resultados apresentados no item anterior, já era de se esperar que as diferenças apresentadas fossem na ordem de um intervalo de $0,50 \mathrm{~m}$ a $2,00 \mathrm{~m}$. No entanto, alguns modelos em alguns pontos apresentaram valores menores, como o AIUB-GRACE03S, que mostrou valor mínimo de $0,07 \mathrm{~m}$ e valor máximo de 1,54 m, apresentando uma amplitude de 1,47 (Figura 9). Já as diferenças entre o GO_CONS_GCF_2_TIM_R2 e o MAPGEO2010, apresentaram valores mínimo e máximo de $\overline{0,32} \mathrm{~m}$ e $1,60 \mathrm{~m}$, respectivamente, com amplitude de $1,27 \mathrm{~m}$ (Figura 10).

Os MGGs AIUB-GRACE03S e o GO_CONS_GCF_2_TIM_R2 quando comparado com o MAPGEO2010, mostraram-se relativamente melhor que o MAPGEO2010 em termos de consistência nos dados para a região em estudo. 
Figura 9 - Diferenças entre o MAPGEO2010 e AIUB-GRACE03S.

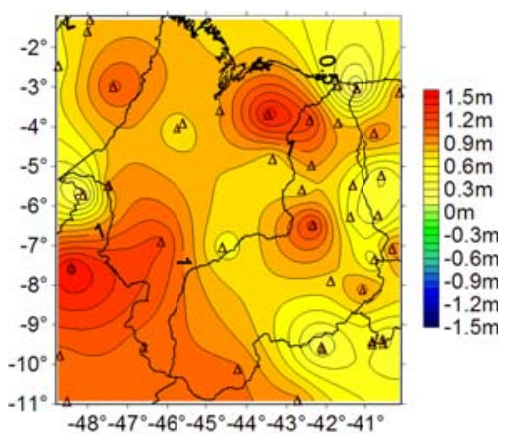

Figura 10 - Diferenças entre o MAPGEO2010 e

GO_CONS_GCF_2_TIM_R2.

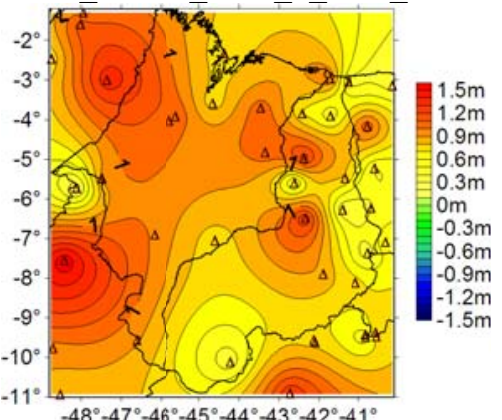

De forma semelhante, avaliaram-se as diferenças do EGM2008 nos seus diferentes graus de desenvolvimento. Para a região em estudo, o EGM2008 atende bem as necessidades dos usuários do sistema de altitudes. Sendo um dos modelos que melhor responde as expectativas da pesquisa, como revelado na avaliação da seção anterior.

As variações das diferenças do EGM2008, nos três graus, em relação ao MAPGEO2010, estão dentro do intervalo de $0,40 \mathrm{~m}$ a $1,60 \mathrm{~m}$. No grau 360 , o modelo revelou resultados das diferenças entre o MGG e o MAPGEO2010 que variaram em torno de $1 \mathrm{~m}$, sendo o valor mínimo apresentado de $0,45 \mathrm{~m}$ e valor máximo de $1,33 \mathrm{~m}$ com amplitude de $0,88 \mathrm{~m}$. Quando se avalia o EGM2008 truncado no grau 720, percebe-se que os valores mínimo e máximo apresentados na avaliação foram de $0,52 \mathrm{~m}$ e 1,46 m, respectivamente, com amplitude de 0,94 m. Já no grau máximo (2190) as discrepâncias encontram-se na faixa de $0,80 \mathrm{~m}$, com valor mínimo de $0,47 \mathrm{~m}$ e valor máximo de 1,46 m com amplitude de 0,99 m (Figura 11).

Para o EGM2008 esperava-se uma menor discrepância, pelo o fato do MAPGEO2010 conter o modelo integrado na sua obtenção, o que implica ter a mesma base de dados relativa à gravimetria, assim como os dados da topografia (MDEs). No entanto, verificou-se uma discrepância equivalente aos outros MGGs quando avaliados em relação ao MAPGEO2010.

O MGG EIGEN05C mais uma vez apresentou um dos resultados mais impactantes. As diferenças com o MAPGEO2010 foram as que mostraram resultados mais destoantes entre os modelos avaliados. Os valores das diferenças comportaram-se de um modo não esperado, apresentou o valor mínimo de $0,11 \mathrm{~m} \mathrm{e}$ o valor máximo de 2,26 m, com amplitude de 2,15 m (Figura 12). 
Figura 11 - Diferenças entre o

MAPGEO2010 e EGM2008 no grau máximo (2190).

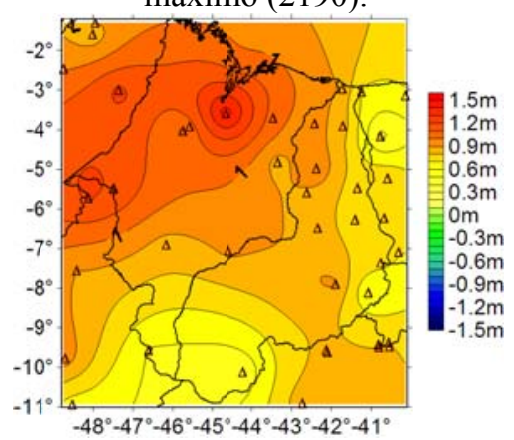

Figura 12 - Diferenças entre o MAPGEO2010 e EIGEN05C.

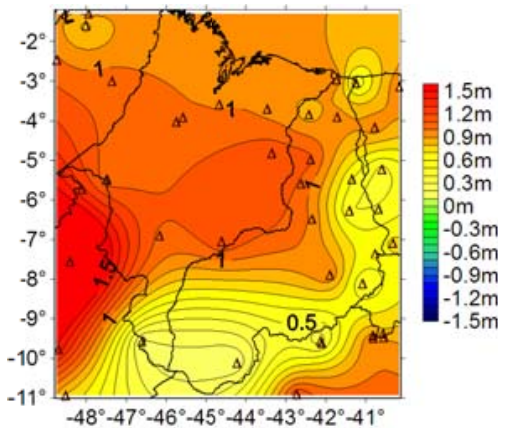

O modelo MAPGEO2010, apesar de ser um modelo desenvolvido com dados de gravimetria terrestre, utilizando o EGM2008 desenvolvido até o grau e ordem 155, o MDE SAM3s_v2, o qual baseia-se no SRTM, com resolução espacial de 3", entre outros dados, ainda apresenta deficiências. A região de estudo apresenta poucas informações geodésicas e os valores de gravidade na região, acredita-se que, apresentam inconsistências.

Um dos maiores problemas associados com possíveis erros de comissão nestes modelos está associado aos possíveis referenciais diferentes aos quais os dados são vinculados. Heck (1990) aborda a geração de distorções nos longos comprimentos de onda nas anomalias de gravidade terrestre causadas pela utilização de diferentes data verticais geodésicos. Assim, além dos erros inerentes dos modelos (comissão e omissão) possam existir erros relacionados à inconsistências de referenciais. Por exemplo, pode existir inconsistência no referencial utilizado na obtenção das anomalias da gravidade com aquele do MDE.

\subsection{Avaliações Relativas}

Na pesquisa realizada por Souza (2006), eventuais distorções da RAFB são avaliadas. Em consequência da extensão do Brasil e o número reduzido de marégrafos, a implantação da RAFB foi realizada por meio de linhas, que com distâncias que ultrapassam alguns milhares de quilômetros, que, mesmo seguindo os padrões de controle recomendados para redes altimétricas de referência, os erros sistemáticos, grosseiros e aleatórios são inevitáveis. Esses erros geram distorções e, consequentemente, deterioram a precisão da rede altimétrica. Também, não existem correções gravimétricas reais na RAFB, conforme já abordado. O posicionamento por GPS, combinado com um modelo geoidal de alta precisão em algumas regiões brasileiras, permitem detectar algumas dessas distorções.

Com isso, adotou-se como estratégia para avaliar as distorções na região em estudo a metodologia descrita a seguir. A avaliação de distorções foi realizada de 
forma relativa, onde se empregou as alturas geoidais nas EG - GPS/NIV da rede e as alturas geoidais advindas dos MGGs e MAPGEO2010.

$\mathrm{Na}$ sequência, apresenta-se a metodologia para uma estação, sendo que a metodologia evidenciada para a estação 1 foi empregada para todas as 20 estações com todos os MGGs empregados no estudo aqui descrito. Para todas as avaliações foram consideradas as alturas geoidais nas EG - GPS/NIV como valor de referência. É importante deixar claro que foram usados 19 pares para cada modelo, totalizando 133 pares de estações.

$\mathrm{Na}$ primeira etapa da avaliação selecionou-se na área de estudo $20 \mathrm{EG}-$ GPS/NIV (Figura 13), das 47 utilizadas na pesquisa. Na sequência calculou-se a diferença entre a altura geoidal da primeira EG - GPS/NIV em relação às outras 19 EG - GPS/NIV, por:

$$
\Delta N_{G P S / N I V}=N_{G P S / N I V}^{1}-N_{G P S / N I V}^{i}
$$

onde $\triangle N_{G P S / N I V}$ é a componente residual entre as alturas geoidais GPS/NIV dos pontos das estações geodésicas GPS/NIV, $N_{G P S / N I V}^{i}$ refere-se à altura geoidal da estação $(i=2,3,4, \ldots, 20)$, obtida pela (02).

Figura 13- Distribuição geográfica das 20 EG - GPS/NIV.

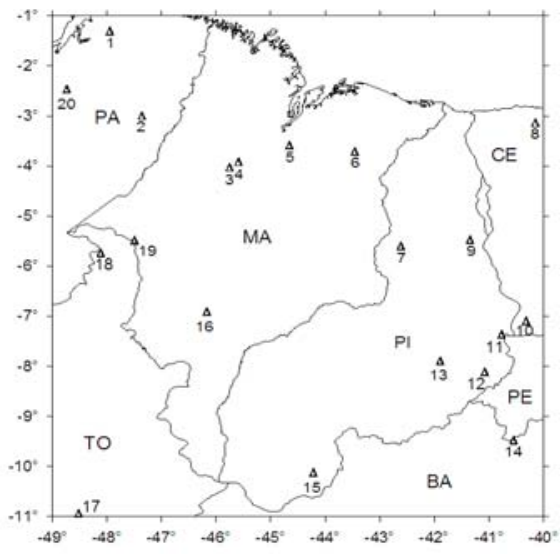

$\mathrm{Na}$ etapa seguinte calculou-se também a diferença entre a altura geoidal da primeira EG - GPS/NIV em relação às 19 dos MGGs e MAPGEO2010, da mesma forma que se calculou na etapa anterior, denominando-se $\Delta N_{M}$.

Posteriormente foram feitas as diferenças entre os resultados das duas etapas anteriores para em seguida calcular o erro relativo, que foi encontrado com a diferença entre as alturas geoidais dividido pela distância entre os pontos. 


$$
\varepsilon_{1}=\frac{\left(\Delta N_{G P S / N I V}-\Delta N_{M}\right)}{D_{i}}
$$

onde $\varepsilon_{l}$ é o erro relativo da primeira EG - GPS/NIV e $D_{i}$ corresponde à distância da primeira estação EG - GPS/NIV entre as 19 estações $(i=2,3,4, \ldots, 20)$.

Ao final de cada avaliação relativa, de cada estação, extraiu-se a Média e o RMS, para obter o erro relativo médio de cada estação. Esta metodologia foi aplicada nas $20 \mathrm{EG}$ - GPS/NIV de cada um dos modelos trabalhados na pesquisa.

Os resultados referentes à avaliação de distorção são os que expressam a aderência das alturas geoidais entre as EG - GPS/NIV e os MGGs, para esta avaliação extraiu-se a média dos valores encontrados e o RMS para cada modelo obtendo o resultado em partes por milhão (ppm).

Os MGGs quando analisados relativamente aos pontos das EG - GPS/NIV, apresentam resultados satisfatórios, dentre eles destaca-se o MGG EGM2008 truncado no grau 360, seguindo do EGM2008 truncado no grau 720 apresentaram os menores valores de RMS e média para a avaliação de distorção e com maior RMS e média os modelos de satélite-somente o AIUB-GRACE03S junto com o GO_CONS_GCF_2_TIM_R2, conforme a Tabela 3.

Tabela 3 - Resumo geral das avaliações de distorções.

\begin{tabular}{l|r|r}
\hline \multicolumn{3}{c}{ Resumo das Médias Gerais } \\
\hline \multirow{2}{*}{ Análise Relativa } & \multicolumn{1}{c}{ Média } & \multicolumn{1}{c}{ RMS } \\
\cline { 2 - 3 } & \multicolumn{2}{c}{$(\mathrm{ppm})$} \\
\hline GO_CONS_GCF_2_TIM_R2 & 0,83 & 1,17 \\
\hline AIUB-GRACE03S & 1,04 & 1,39 \\
\hline EGM2008_2190 & 0,60 & 0,80 \\
\hline EGM2008_720 & 0,59 & 0,76 \\
\hline EGM2008_360 & 0,50 & 0,65 \\
\hline EIGEN_05C & 0,62 & 0,84 \\
\hline MAPGEO2010 & 0,75 & 0,86 \\
\hline
\end{tabular}

Cabe ressaltar que o EGM2008 desenvolvido até o grau máximo, que tem uma resolução espacial de aproximadamente $9 \mathrm{~km}$, também apresentou bom desempenho para esta análise, sendo que para os valores de RMS e média o modelo demonstrou resultados bastante similares em seus três graus trabalhados na pesquisa.

É importante salientar que todos os modelos analisados, apresentam bom desempenho relativo. O modelo AIUB_GRACE_03S é o que apresenta desempenho 
relativo com RMS mais elevado, comparado com os demais, porém não pode ser classificado como insatisfatório.

Na Tabela 4 ilustram-se síntese dos resultados individuais das médias e RMS das avaliações de distorções, para cada uma as 4 primeiras das 20 estações em todos os modelos trabalhados. A análise completa de todos os pares podem ser conferidas em Melo (2011).

Tabela 4 - Síntese das avaliações de distorções das estações 1 a 4.

\begin{tabular}{l|c|c|c|c|c|c|c|c|c}
\hline \multirow{2}{*}{ Estações } & \multicolumn{2}{c|}{$\mathbf{1}$} & \multicolumn{2}{c|}{$\mathbf{2}$} & \multicolumn{2}{c}{3} & \multicolumn{2}{c}{4} \\
\hline \multirow{2}{*}{ Modelos } & Méd. & RMS & Méd. & RMS & Méd. & RMS & Méd. & RMS \\
\cline { 2 - 9 } & \multicolumn{7}{c}{ (ppm) } \\
\hline GOCE_TIM_R2 & 0,68 & 0,87 & 0,73 & 1,25 & 0,94 & 1,48 & 1,12 & 1,75 \\
\hline $\begin{array}{l}\text { AIUB- } \\
\text { GRACE03S }\end{array}$ & 0,88 & 0,97 & 0,80 & 1,12 & 1,02 & 1,36 & 1,02 & 1,33 \\
\hline EGM2008_2190 & 0,76 & 0,86 & 0,68 & 0,84 & 0,74 & 1,14 & 0,73 & 1,14 \\
\hline EGM2008_720 & 0,76 & 0,85 & 0,70 & 0,85 & 0,71 & 1,05 & 0,70 & 1,06 \\
\hline EGM2008_360 & 0,73 & 0,80 & 0,76 & 0,93 & 0,46 & 0,70 & 0,46 & 0,69 \\
\hline EIGEN_05C & 0,63 & 0,71 & 0,81 & 0,98 & 0,60 & 0,82 & 0,60 & 0,83 \\
\hline MAPGEO2010 & 0,67 & 0,71 & 1,00 & 1,09 & 0,71 & 0,86 & 0,73 & 0,89 \\
\hline
\end{tabular}

\section{CONCLUSÕES}

Foi atingido o objetivo central deste trabalho de avaliar os modelos globais do geopotencial disponibilizados atualmente pelo ICGEM, via comparações com as alturas geoidais das EG - GPS/NIV do SGB. O experimento foi efetivado sobre um total de 2 modelos satélite somente AIUB-GRACE03S e GO_CONS_GCF_2_TIM_R2 e 2 modelos combinados EINGEN-05C e EGM2008 (360, 720 e 2190) além do modelo adaptado MAPGEO2010. Dos resultados apresentados cabe enfatizar alguns aspectos importantes, conforme abordado na sequência.

Foram efetivadas as avaliações do desempenho dos MGGs satélite-somente e combinados mais atuais e preconizados com tendo os menores erros de comissão. Os erros de omissão referem-se exclusivamente ao seu grau de desenvolvimento harmônico. Avaliou-se o modelo adaptado MAPGEO2010 pelo seu caráter oficial e também em vista de algumas evidências prévias de sua falta de adequação à região de estudos, quando avaliado do ponto de vista absoluto. Utilizou-se como referência fundamental as observações da altitude elipsoidal com GPS sobre referências de nível da RAFB, dados estes obtidos do banco de dados do SGB/IBGE. 
O aspecto mais marcante das avaliações foi o de que os MGGs analisados com os maiores erros de omissão, surpreendentemente, não foram os de pior desempenho nas análises tanto do ponto de vista absoluto quanto relativo.

Todos os MGGs tiveram tendências sistemáticas similares na região com valor médio de 0,336 m. Já o MAPGEO2010, de acordo com as análises efetivadas, apresentou tendência sistemática de $-0,52 \mathrm{~m}$, mostrando menor aderência com a estrutura geodésica na região de estudos. O modelo MAPGEO2010, que deveria ser o de menor erro de omissão por ser composto de diversos dados à área de estudos, não tem bom desempenho absoluto na região. Possui a maior tendência sistemática e juntamente com o modelo EIGEN-05C apresentam os piores RMS.

O EGM2008 apresentou um bom compromisso entre resolução, acurácias absoluta e relativa e tendência sistemática, em seus três graus $(360,720$ e 2190) de desenvolvimento da pesquisa.

Todos os modelos analisados, inclusive o MAPGEO2010, eliminadas as tendências sistemáticas, apresentam bom desempenho relativo. Mesmo o modelo AIUB_GRACE_02S que apresenta desempenho relativo com RMS mais elevado não pode ser classificado como inadequado. Assim, recomenda-se que sempre a aplicação do modelo geoidal seja procedida na forma relativa para a região de estudos.

O MAPGEO2010 no ponto de vista absoluto apresentou o pior desempenho, isso está ligado a uma série de inconsistências que o modelo tem na região de estudo, provavelmente associada aos valores de gravidade no local, inconsistências de referenciais e possivelmente no termo de ordem zero empregado na sua obtenção e que talvez já estivesse presente na aplicação do EGM2008.

A tendência sistemática média observada para os MGGs empregados foi de aproximadamente $0,33 \mathrm{~m}$. Valor este similar ao da TNMM determinada preliminarmente para o Datum Vertical Brasileiro em trabalhos anteriores.

O modelo de melhor desempenho na região de estudos, quando das avaliações da componente sistemática via o RMS, adequada acurácia relativa e menor tendência sistemática foi o EGM2008 nos três graus de desenvolvimento. Sendo que nas três suas três resoluções empregadas (360, 720 e 2190) o modelo não apresentou muita diferença nas avaliações.

Mesmo considerado o EGM2008 como de melhor desempenho global na região, tais resultados não devem ser generalizados. O que se observa é que, no estágio atual de desenvolvimento, os MGGs e MAPGEO2010 podem ser cotejados como adequados somente para aplicações de propagação de alturas geoidais na forma relativa.

\section{AGRADECIMENTOS}

Os autores agradecem à CAPES pelo suporte aos estudos do primeiro autor e CNPq, processo 301797/2008-0 pelo apoio à pesquisa. Também agradecem ao IBGE e ICGEM pelo acesso à base de dados e aplicativos utilizados. 


\section{REFERÊNCIAS BIBLIOGRÁFICAS}

BURŠA, M.; KOUBA, J.; KUMAR, M.; MÜLLER, A.; RADĚJ, K.; TRUE, S.A.; VATRT, V.; VOJTÍŠKOVÁ, M. Geoidal geopotential and world height system. Studia Geophysica et Geodaetica, Praga, v. 43, n. 4, p. 327-337, 1999.

DE FREITAS, S. R. C. O papel da Geodésia para a monitoramento das mudanças globais. Palestra - III Simpósio Brasileiro de Ciências Geodésicas e Tecnologia de Geoinformação (SIMGEO), Recife - PE, 2010. www.ufpe.br/cgtg/SIMGEOIII/index1.htm. Anais ISBN: 978-85-63978-00-4

DE FREITAS, S. R. C. ; FERREIRA, V. G.; PALMEIRO, A. S.; DALAZOANA, R.; LUZ, R. T.; FAGGION, P. L. Modelagem do potencial anômalo no Datum Vertical Brasileiro visando a sua nova definição. Boletim de Ciências Geodésicas, Curitiba, v. 13, n. 2. p. 395-419, 2007.

DE FREITAS, S. R. C.; FERREIRA, V. G.; PALMEIRO, A. S.; CARVALHO, J. L. B. de; SILVA, L. F. Analysis of the geopotential anomalous component at Brazilian Vertical Datum region based on the imarui lagoon system. Gravity, Geoid and Earth Observation, International Association of Geodesy Symposia 135, chapter 42, DOI 10.1007/978-3-642-10624-7, S. P. Mertikas (ed.) Springer-Verlag Berlin Heidelberg. 2010.

DRINKWATER, M. R. et al, (2007). The GOCE Gravity Mission: ESA'S First Core Earth Explorer; Proceedings of the $3^{\text {rd }}$ International GOCE User Workshop, 6-8 November, 2006, Frascati, Italy, ESA Special Publication, SP627, ISBN 92-9092-938-3, pp.1-8, 2007.

FERREIRA, V. G. Análise da componente anômala do geopotencial no Datum Vertical Brasileiro com base no Sistema Lagunar de Imarui, SC. Dissertação Curso de Pós-Graduação em Ciências Geodésicas, Setor de Ciências da Terra, Universidade Federal do Paraná. Curitiba - PR. 115pp. 2008.

FERREIRA, V. G.; DE FREITAS, S. R. C.; HECK, B. A separação entre o geoide e o quase geoide: uma análise no contexto brasileiro. Revista Brasileira de Cartografia Nº 63 Edição Especial 40 Anos, 2011. (ISSN 0560-4613). 2011.

GEMAEL, C. Introdução à geodésia física. Curitiba: Editora da UFPR, 2002.

HECK, B. (1990) An evaluation of some systematic error sources affecting terrestrial gravity anomalies. Bull. Géodés., 64, 88-108p. 1990.

HEISKANEN, W. A.; MORITZ, H. (1967) Physical Geodesy. San Francisco: W. H. Freeman, 1967.

HEISKANEN, W. A.; MORITZ, H. (1979) Physical Geodesy. Austria: Reprint Institute of Physical Geodesy, Technical University, 1979.

IBGE (2011a). Instituto Brasileiro de Geografia e Estatística: Modelo de Ondulação Geoidal: Introdução, Cálculo e Avaliação do Modelo. Disponível em: <http://www.ibge.gov.br/home/geociencias/geodesia/ modelo_geoidal. shtm> Acessado em 2011.

IBGE (2011b). Instituto Brasileiro de Geografia e Estatística: SGB - Sistema Geodésico Brasileiro: Introdução. Disponível em: <http://www.ibge. gov.br/home/geociencias/geodesia/default_sgb_int.shtm> Acessado em 2011. 
ICGEM (2010). International Centre for Global Earth Models: Global Gravity Field Models. Disponível em: <http://icgem.gfz-potsdam.de /ICGEM /ICGEM.html > Acessado em 2010.

JAMUR, K. P; DE FREITAS, S. R. . Estudo comparativo dos modelos gravimétricos globais eigengl04c, eigen-cg03c e eigen-05c utilizando um Modelo Regional e dados gps/niv para o estado do Paraná. Boletim Ciências Geodésicas, sec. Artigos, Curitiba, v. 15, n 4, p.527-543, out-dez, 2009.

JEKELI, C. Heights, geopotential, and vertical data. Technical Report n. 459, OSU. 2000.

KAULA, W.M. Theory of Satellite Geodesy: Applications of satellites to Geodesy. Re-published by Dove ed. 124pp. Original by Blaisdell Publishing Co., 1966.

LEMOINE, F. et al. The deveploment of the joint NASA - GSFC and the NIMA Geopotential Model EGM 96. NASA/TP - 1998 - 206861.

PALMEIRO, A. S. Análise do vínculo do DVB a um Sistema Global de Altitudes com base em soluções fixadas e livres do PVCG. Tese de doutorado, CPGCG, UFPR, 144pp., 2011.

PAVLIS, N. K., HOLMES S. A., KENYON S. C., FACTOR J. K. An Earth Gravitational Model to Degree 2160: EGM2008; presented at the 2008 General Assembly of the European Geosciences Union, Vienna, Austria, April 13-18, 2008.

REFAG2010 - Reference Frames for Applications in Geosciences 2010. Definition and establishment of vertical reference systems. Commission 1 Symposium - Session 5, International Association of Geodesy IAG, <http://iag.ign.fr/index.php?id=151> Acessado em setembro de 2011.

SÁNCHEZ, L.; MARTÍNEZ, W. (2008). Avances en el processamiento unificado de las redes verticales involucradas en SIRGAS. In: SIRGAS Meeting, May 28-29, Montevideo, Uruguay. 2008.

SOUZA, D. V. (2006) Avaliação de distorções da rede altimétrica fundamental no sul/sudeste do Brasil usando o geóide e GPS. Dissertação (Mestrado), Departamento de Geofísica, Instituto de Astronomia, Geofísica e Ciências Atmosféricas, Universidade de São Paulo, São Paulo - SP, 124p. 2006.

(Recebido em janeiro de 2012. Aceito em abril de 2012.) 